\title{
Integration of disease-specific single nucleotide polymorphisms, expression quantitative trait loci and coexpression networks reveal novel candidate genes for type 2 diabetes
}

\author{
H. P. Kang $\cdot$ X. Yang $\cdot$ R. Chen $\cdot$ B. Zhang $\cdot$ E. Corona $\cdot$ \\ E. E. Schadt • A. J. Butte
}

Received: 15 December 2011 / Accepted: 2 April 2012 /Published online: 16 May 2012

(C) The Author(s) 2012. This article is published with open access at Springerlink.com

\begin{abstract}
Aims/hypothesis While genome-wide association studies (GWASs) have been successful in identifying novel variants associated with various diseases, it has been much more difficult to determine the biological mechanisms underlying these associations. Expression quantitative trait loci (eQTL) provide another dimension to these data by associating single nucleotide polymorphisms (SNPs) with gene expression. We hypothesised that integrating SNPs known to be associated with type 2 diabetes with eQTLs and coexpression networks would enable the discovery of novel candidate genes for type 2 diabetes.

Methods We selected 32 SNPs associated with type 2 diabetes in two or more independent GWASs. We used previously described eQTLs mapped from genotype and gene expression data collected from 1,008 morbidly obese patients to find genes with expression associated with these SNPs. We linked these genes to coexpression modules, and
\end{abstract}

\footnotetext{
H. P. Kang $\cdot$ R. Chen $\cdot$ E. Corona $\cdot$ A. J. Butte $(\bowtie)$

Division of Systems Medicine, Department of Pediatrics,

Stanford University School of Medicine,

1265 Welch Road, Room X163,

Stanford, CA 94305, USA

e-mail: abutte@stanford.edu
}

H. P. Kang • R. Chen · E. Corona • A. J. Butte

Lucile Packard Children's Hospital,

Palo Alto, CA, USA

X. Yang $\cdot$ B. Zhang

Sage Bionetworks,

Seattle, WA, USA

E. E. Schadt

Department of Genetics and Genome Sciences,

Mount Sinai School of Medicine,

New York, NY, USA ranked the other genes in these modules using an inverse sum score.

Results We found 62 genes with expression associated with type 2 diabetes SNPs. We validated our method by linking highly ranked genes in the coexpression modules back to SNPs through a combined eQTL dataset. We showed that the eQTLs highlighted by this method are significantly enriched for association with type 2 diabetes in data from the Wellcome Trust Case Control Consortium (WTCCC, $p=0.026)$ and the Gene Environment Association Studies (GENEVA, $p=0.042$ ), validating our approach. Many of the highly ranked genes are also involved in the regulation or metabolism of insulin, glucose or lipids.

Conclusions/interpretation We have devised a novel method, involving the integration of datasets of different modalities, to discover novel candidate genes for type 2 diabetes.

Keywords Genetics of type 2 diabetes .

Genomics/proteomics $\cdot$ Mathematical modelling and simulation

$\begin{array}{ll}\text { Abbreviations } \\ \text { eQTL } & \text { Expression quantitative trait locus } \\ \text { eSNP } & \text { Expression single nucleotide polymorphism } \\ \text { GENEVA } & \text { Gene-Environment Association Studies } \\ \text { GWAS } & \text { Genome-wide association study } \\ \text { LD } & \text { Linkage disequilibrium } \\ \text { SNP } & \text { Single nucleotide polymorphism } \\ \text { WTCCC } & \text { Wellcome Trust Case Control Consortium }\end{array}$

\section{Introduction}

Genome-wide association studies (GWASs) of common complex or multifactorial diseases have proliferated enormously 
over the last few years. They have also been successful in identifying a large number of loci at extraordinary levels of significance, given the large cohort sizes. However, this success has presented a new challenge: translating these findings into a full understanding of how the loci affect complex disease traits. Most of the reported variants do not affect protein function in an obvious manner and indeed a large number lie in introns or intergenic regions [1], indicating that they may function through other regulatory mechanisms such as control of gene expression or alternative splicing. Even the gene or genes implicated in a given disease with respect to a given genetic locus in such cases is not entirely clear, given that variation near one gene may affect regulation of another neighbouring gene, or variation in the intron of a proteincoding gene may affect the regulation of an embedded noncoding RNA. Variations in DNA affecting the expression of a gene begs the question of whether such genetic variants, commonly known as expression quantitative trait loci (eQTLs), are an important factor in disease susceptibility [2].

The genes regulated by eQTLs are commonly referred to as expression traits. The relationship between eQTL genotypes and expression traits are mapped by performing GWASs for the expression of all genes in the organism of interest [3]. This requires simultaneous measurement of genetic variation (single nucleotide polymorphisms [SNPs]) and gene expression in the same individuals. Multiple hypothesis correction, either through applying a Bonferroniadjusted cut-off or more sophisticated methods such as permutation-based analysis to empirically estimate the null distribution and control the false discovery rate (FDR), is then used to filter the association results. The SNPs that tag eQTLs are commonly referred to as expression SNPs (eSNPs). However, eQTL-expression trait relationships are not just statistical associations. Nicolae et al demonstrated that trait-associated SNPs in general are enriched for eQTLs [4]. In addition, studies have shown that eQTLs mapped in a disease-relevant tissue of interest are enriched for diseaseassociated SNPs [5, 6]. These insights have been applied to leverage eQTLs in providing a more relevant context within which to interpret SNP associations to disease and to prioritise GWAS results [6-8]. Other investigators have taken advantage of the link between genetic variation and gene expression by integrating eQTLs with coexpression networks and showing that this is a powerful model for discovering novel genes and gene networks relevant to disease $[9,10]$.

In this study, we demonstrate a novel approach integrating eQTLs and mouse tissue-specific coexpression networks with knowledge of genetic variants reproducibly associated with type 2 diabetes. Our hypothesis was that if the genes coexpressed with expression traits linked to the well-known type 2 diabetes SNPs have any explanatory power or biological relevance for type 2 diabetes, then eSNPs linked with expression traits for these coexpressed genes should already have been associated with type 2 diabetes in previously run case-control GWASs, albeit at a much lower, indirect level of significance. Showing weaker but consistent levels of significance in case-control studies for these other eSNPs would potentially serve as a valuable method for explaining some of the 'missing heritability' in the genetic architecture of complex disease, while at the same time serving as a method to explain those variants already discovered in GWASs.

\section{Methods}

The overall experimental design is shown in Fig. 1. We first constructed a comprehensive dataset of eQTLs discovered in metabolically significant tissues by collecting all SNP-gene expression relationships with genome-wide significance reported in liver and subcutaneous and omental adipose tissue $[10,11]$.

To identify a starting set of genes associated with type 2 diabetes, we first selected SNPs that had been reproducibly associated with type 2 diabetes in two or more independent GWASs. To map these type-2-diabetes-associated SNPs to candidate causal genes, we used cis SNP-gene associations (gene to SNP distance within $1 \mathrm{Mb}$ ) identified with the Kruskal-Wallis test at an uncorrected $p$ value cut-off of 0.05 in liver and subcutaneous and omental adipose tissue of 1,008 morbidly obese patients [10]. In total, there were 508 genes within $1 \mathrm{Mb}$ of these SNPs. We decided that the higher false-positive rate resulting from this relaxed cut-off could be tolerated because we planned to use additional filters to genetically validate any novel associations. We refer to these type 2 diabetes candidate genes mapped via eQTLs as type 2 diabetes expression traits.

We obtained previously described tissue-specific coexpression subnetworks constructed from gene expression studies in human tissues $[10,12,13]$ and various mouse crosses, including $\mathrm{C} 57 \mathrm{BL} 6 / \mathrm{J} \times \mathrm{C} 3 \mathrm{H} / \mathrm{HeJ}$ on both wild type [11] and $\mathrm{Apoe}^{-/-}$backgrounds [9, 14], C57BL6/J $\times \mathrm{A} / \mathrm{J}[15]$ and C57BL6/J $\times$ Castaneus [11]. The weighted coexpression subnetworks were constructed with previously described methods using the most highly connected nodes from each tissue [16]. Briefly, the networks are based only on calculated gene-gene pairwise correlations, with the modules in these networks identified based on topological properties in an approach that avoids hard thresholding on the correlations. The weighted network analysis begins with a matrix of the Pearson correlations between all gene pairs and then converts the correlation matrix into an adjacency matrix using a power function $f(x)=x^{b}$. The variable $b$ of the power function is determined in such a way that the resulting adjacency matrix, namely the weighted coexpression 


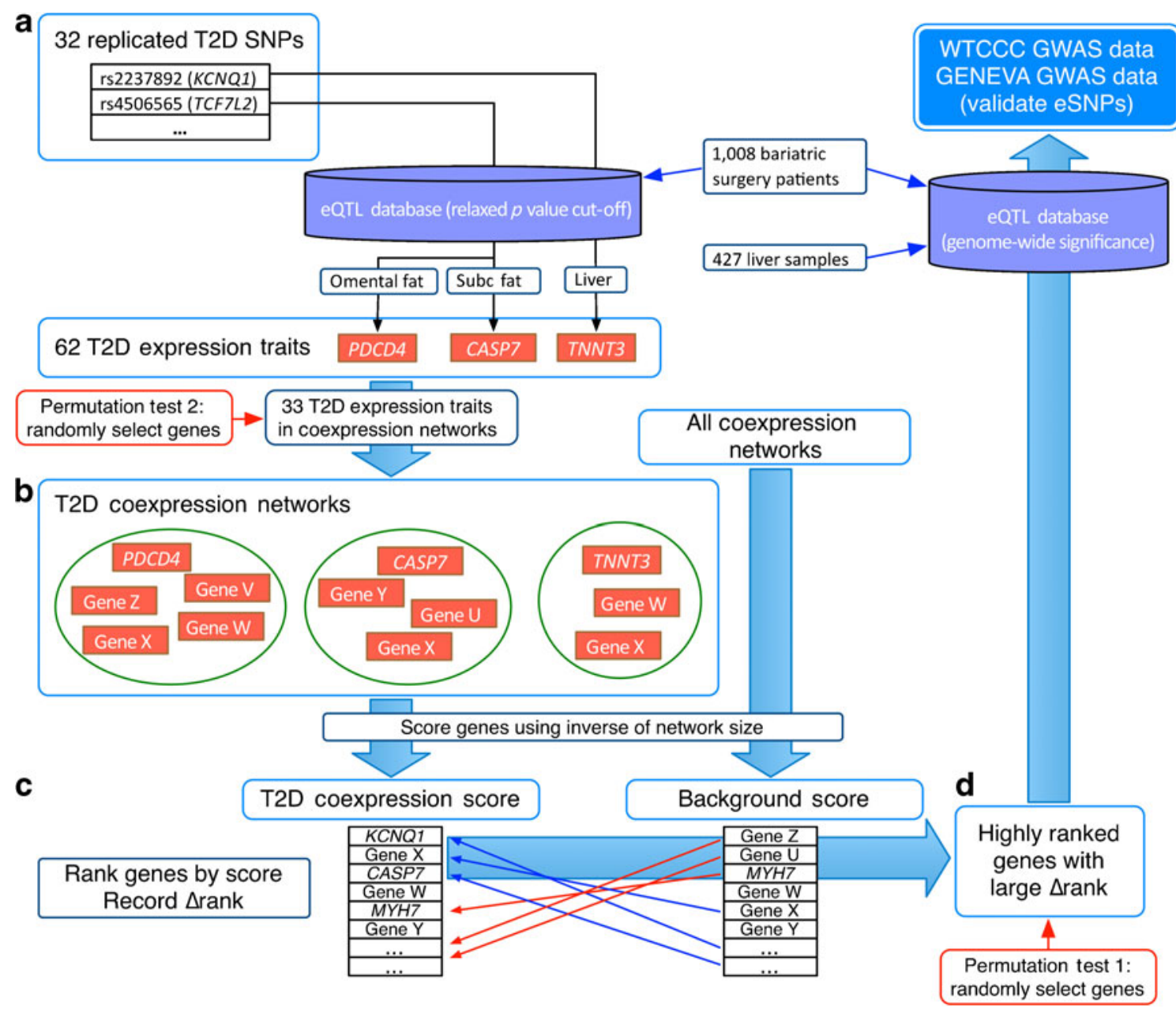

Fig. 1 Integrating replicated disease SNPs with differentially expressed genes through an eQTL dataset. (a) We mapped 33 SNPs replicated in more than one GWAS for type 2 diabetes to 62 expression traits in liver and omental and subcutaneous adipose tissue taken from morbidly obese individuals. (b) There were 526 coexpression networks that contained at least one of these expression traits. (c) The score for each gene in the coexpression networks was calculated by taking the

network, is approximately scale-free. By not including literature, canonical pathways and other such relationships among genes, the coexpression networks represent a more unbiased view of the biological processes (reflected in the modules) at play.

Subnetworks that contained at least one type 2 diabetes expression trait were selected; these will be referred to as type 2 diabetes coexpression networks. Genes that appeared in more of these modules could be considered more likely to be associated with type 2 diabetes. We therefore scored genes for type 2 diabetes coexpression by counting the number of modules they appeared in. Because a gene would be more likely to be present in a larger module simply by chance, we weighted the score by taking the inverse of the network size. Therefore, the type 2 diabetes coexpression score for each gene was calculated by summing the inverse sum of the inverse of the network sizes in which it appeared. A background score was also calculated for all coexpression networks, then the two sets of genes were ranked by score and the $\Delta$ rank was recorded. (d) Highly ranked genes with large $\Delta$ rank were mapped back to eSNPs, and we evaluated the distribution of $p$ values of these eSNPs in data from the WTCCC. The two different models for the permutations are also shown. Subc, subcutaneous; T2D, type 2 diabetes

of the number of genes in each type 2 diabetes coexpression network containing that gene, or $\sum_{i}^{n} 1 / S_{i}$, where $n$ is the number of networks in which the gene co-occurs with an expression trait and $S_{i}$ is the number of genes in network $i$. The background score was calculated by repeating this analysis for the genes in all coexpression networks.

Genes were mapped back to eSNPs using the previously mentioned eQTL dataset containing SNP-RNA relationships of genome-wide significance. To test whether the eSNPs of genes above a certain score cut-off were enriched for association to type 2 diabetes, we used SNP summary statistics provided by the Wellcome Trust Case Control Consortium (WTCCC). Because the eQTL mapping studies and the WTCCC assayed SNPs on different platforms with incomplete overlap, the eSNPs were expanded to include those in strong linkage disequilibrium (LD; $\left.R^{2}>0.8\right)$. The 
percentage with $p<0.05$ for type 2 diabetes $\left(p_{\mathrm{T} 2 \mathrm{D}}<0.05\right)$ was recorded. Because we would expect type 2 diabetes expression traits and genes containing type 2 diabetes SNPs to score highly using our filters, we removed these from the novel candidate gene lists before performing this evaluation.

We empirically estimated the null distribution of eSNPs using two different models. For the first model, we randomly selected half of the genes above a certain score cut-off, mapped these to eSNPs, expanded into strong LD, and recorded $p_{\text {T2D }}<0.05$ as above. This was repeated 10,000 times for each cut-off. For the second model, we randomly selected 33 genes as the initial expression traits used to determine the subnetworks and repeated the entire analysis. This process was repeated 1,000 times.

We also used an independent GWAS dataset from the Gene-Environment Association Studies (GENEVA) Diabetes Study (release Version 2) to validate our findings. This is a GWAS performed on a total of 3,000 cases and 3,000 controls from two well-characterised cohorts: the Nurses' Health Study (www.channing.harvard.edu/nhs) and the Health Professionals Follow-up Study (www.hsph.harvard. edu/hpfs). We obtained SNP summary statistics through the Database of Genotypes and Phenotypes (dbGaP; dbGaP Study Accession: phs000091.v2.p1) and performed the same permutation analysis by randomly selecting 33 genes as the initial expression traits.

\section{Results}

Type 2 diabetes expression traits We started with 32 SNPs that have been associated with type 2 diabetes in multiple studies (Table 1) [17-22]. Using genotype data of 1,008 patients and gene expression profiles of three tissues (omental adipose, subcutaneous adipose and liver) collected at the time of gastric bypass surgery [10], we identified genes within $1 \mathrm{Mb}$ of each type 2 diabetes SNP with expression correlated with the genotype at that locus. In total, only 21 of these 32 type 2 diabetes SNPs were associated with 62 different expression traits at the uncorrected threshold of $p=0.05$.

Scoring genes using mouse coexpression networks In order to study these 62 expression traits associated with 21 wellknown type 2 diabetes SNPs, we screened through 2,326 tissue-specific coexpression modules derived from coexpression networks constructed as previously described [9-16]. Out of the 62 type 2 diabetes expression traits, 33 were present in one or more coexpression network modules, resulting in the implication of 526 type 2 diabetes coexpression network modules.

Each of the 13,961 genes that were present in any of these 526 networks could contribute additional explanatory power as to how those original 21 type 2 diabetes SNPs lead
Table 1 Thirty-two SNPs reproducibly associated with type 2 diabetes in multiple studies

\begin{tabular}{|c|c|c|c|}
\hline SNP & Chromosome & Functional type & Adjacent gene \\
\hline rs $7578597^{\mathrm{a}}$ & 2 & Missense & THADA \\
\hline $\mathrm{rs} 4402960^{\mathrm{a}}$ & 3 & Intron & $I G F 2 B P 2$ \\
\hline rs $1470579^{a}$ & 3 & Intron & $I G F 2 B P 2$ \\
\hline rs $4607103^{a}$ & 3 & Intergenic & \\
\hline rs $10010131^{\mathrm{a}}$ & 4 & Intron & WFS1 \\
\hline rs 7754840 & 6 & Intron & $C D K A L 1$ \\
\hline rs7756992 & 6 & Intron & $C D K A L 1$ \\
\hline rs10946398 & 6 & Intron & $C D K A L 1$ \\
\hline rs4712523 & 6 & Intron & $C D K A L 1$ \\
\hline rs $864745^{\mathrm{a}}$ & 7 & Intron & $J A Z F 1$ \\
\hline rs $13266634^{\mathrm{a}}$ & 8 & Missense & SLC $30 A 8$ \\
\hline rs 10811661 & 9 & Intergenic & \\
\hline rs $564398^{\mathrm{a}}$ & 9 & Intergenic & \\
\hline rs $7903146^{\mathrm{a}}$ & 10 & Intron & $T C F 7 L 2$ \\
\hline rs $12255372^{\mathrm{a}}$ & 10 & Intron & $T C F 7 L 2$ \\
\hline rs $7901695^{\mathrm{a}}$ & 10 & Intron & $T C F 7 L 2$ \\
\hline rs $11196205^{\mathrm{a}}$ & 10 & Intron & $T C F 7 L 2$ \\
\hline rs $7895340^{\mathrm{a}}$ & 10 & Intron & $T C F 7 L 2$ \\
\hline $\operatorname{rs} 4506565^{\mathrm{a}}$ & 10 & Intron & $T C F 7 L 2$ \\
\hline $\mathrm{rs} 1111875^{\mathrm{a}}$ & 10 & Intergenic & HHEX \\
\hline $\mathrm{rs} 5015480^{\mathrm{a}}$ & 10 & Intergenic & HHEX \\
\hline rs $12779790^{\mathrm{a}}$ & 10 & Intergenic & \\
\hline rs5219 & 11 & Missense & KCNJ11 \\
\hline rs $2237892^{a}$ & 11 & Intron & $K C N Q 1$ \\
\hline rs2237895 & 11 & Intron & $K C N Q 1$ \\
\hline $\operatorname{rs} 2237897^{\mathrm{a}}$ & 11 & Intron & $K C N Q 1$ \\
\hline rs10830963 & 11 & Intron & MTNRIB \\
\hline rs7961581 ${ }^{a}$ & 12 & Intergenic & \\
\hline rs8050136 & 16 & Intron & FTO \\
\hline rs9939609 & 16 & Intron & FTO \\
\hline rs4430796 & 17 & Intron & $H N F 1 B$ \\
\hline rs1884613 & 20 & Intergenic & \\
\hline
\end{tabular}

${ }^{\text {a }}$ Serves as an eSNP in liver and adipose tissue

to diabetes. To study each of these 13,961 genes, we calculated the type 2 diabetes coexpression score as $\sum_{i}^{n} 1 / S_{i}$, where $n$ is the number of networks in which the gene is coexpressed with one of the 33 expression traits and $S_{i}$ is the total number of genes in network $i$. In order to determine a baseline or background score, we treated the entire set of 2,326 coexpression modules as describing all human disease (disease coexpression networks) and calculated the score for all 13,987 genes that appeared in any of these 2,326 coexpression modules. This was done to control for genes present in a high percentage of all networks, for example. We then ranked genes in the type 2 diabetes and background coexpression score lists from highest to lowest scores and 
recorded the $\Delta$ rank ([Background coexpression rank]-[Type 2 diabetes coexpression rank]) for each gene, resulting in a type 2 diabetes coexpression rank and $\Delta$ rank for each gene. The rank and $\Delta$ rank for the type 2 diabetes expression traits are listed in Table 2.

eSNPs of highly ranked genes are enriched for association with type 2 diabetes As stated above, our hypothesis was that if the genes coexpressed with expression traits linked to the well-known type 2 diabetes SNPs have any explanatory power or biological relevance for type 2 diabetes, then variants in these genes might have already been associated with type 2 diabetes in case-control studies, albeit at a much lower, indirect, level of significance. We thus evaluated the performance of the type 2 diabetes coexpression score and rank change for selecting genes relevant to the pathogenesis of type 2 diabetes. Of the 13,961 genes coexpressed with the expression traits of type 2 diabetes SNPs, 6,030 (6,005 excluding type 2 diabetes expression traits) could be mapped back through eQTLs to 20,480 SNPs that had been tested in the well-known GWAS on type 2 diabetes, run by the WTCCC.

We selected sets of these 6,005 genes at six type 2 diabetes coexpression rank quantile cut-offs $(0,75,90,95$, 97.5, 99). Although there was a trend for increasing enrichment of WTCCC $p_{\mathrm{T} 2 \mathrm{D}}<0.05$ in the higher quantiles, it was not significant. Because we expected genes relevant to type 2 diabetes to have a high $\Delta$ rank compared with background, we ordered each quantile by $\Delta$ rank, then split each set into those above and below the median $\Delta$ rank for that quantile. We compared the WTCCC $p_{\mathrm{T} 2 \mathrm{D}}<0.05$ of the subsets of genes above and below the median $\Delta$ rank (Fig. 2a), revealing the genes with high $\Delta$ rank in each set to be increasingly enriched for WTCCC $p_{\mathrm{T} 2 \mathrm{D}}<0.05$ at higher cut-offs and thus confirming our original hypothesis.

The null distributions for the halves of each quantile were estimated by randomly selecting half of the genes in each set and recording the $p_{\mathrm{T} 2 \mathrm{D}}<0.05$. This was repeated 10,000 times. This analysis revealed that the enrichment trend observed in Fig. 2a reached significance at the 99th percentile cut-off $(18.7 \%, p=0.007)$. We also performed a more stringent estimation of the null distribution by randomly selecting 33 genes as the initial expression traits and replicating the analysis 1,000 times. The result of permutation using this model was consistent with the first one, with a $p$ value of 0.026 at the 99th percentile cut-off (Fig. 2b). In order to validate these findings, we repeated the analysis using SNP data from the GENEVA diabetes study. A total of 7,047 genes (7,037 excluding type 2 diabetes expression traits) could be mapped back through eQTLs to 31,011 SNPs tested in this study. Similar to what we had seen with WTCCC, we observed significant enrichment $(19.5 \%$ in the 99.75 th percentile, $p=0.042$ ) for GENEVA $p_{\mathrm{T} 2 \mathrm{D}}<0.05$ at higher cut-offs.
Table 2 Thirty-three expression traits regulated by type 2 diabetes SNPs

\begin{tabular}{|c|c|c|c|c|}
\hline SNP & $\begin{array}{l}\text { Adjacent } \\
\text { gene }\end{array}$ & $\begin{array}{l}\text { Expression } \\
\text { trait }\end{array}$ & $\begin{array}{l}\mathrm{T} 2 \mathrm{D} \\
\text { coexpression } \\
\text { rank }\end{array}$ & $\Delta$ Rank \\
\hline $\begin{array}{l}\text { rs2237892 } \\
\text { rs2237897 }\end{array}$ & $K C N Q 1$ & $K C N Q 1$ & 1 & 654 \\
\hline rs2237892 & $K C N Q 1$ & TNNT3 & 3 & 1,265 \\
\hline $\begin{array}{l}\text { rs } 1111875 \\
\text { rs5015480 }\end{array}$ & & $I D E$ & 23 & 2,508 \\
\hline $\begin{array}{l}\text { rs1111875 } \\
\text { rs5015480 }\end{array}$ & & CYP26A1 & 27 & 2,674 \\
\hline rs4607103 & & ADAMTS9 & 34 & 2,840 \\
\hline rs 12255372 & $T C F 7 L 2$ & $V T I 1 A$ & 35 & 2,843 \\
\hline $\begin{array}{l}\text { rs } 4506565 \\
\text { rs7895340 }\end{array}$ & TCF7L2 & $C A S P 7$ & 42 & 3,302 \\
\hline \multicolumn{5}{|l|}{ rs11196205 } \\
\hline \multicolumn{5}{|l|}{ rs7901695 } \\
\hline rs 12255372 & $T C F 7 L 2$ & $G P A M$ & 43 & 3,428 \\
\hline $\begin{array}{l}\text { rs5015480 } \\
\text { rs1111875 }\end{array}$ & & PDLIMI & 48 & 3,603 \\
\hline rs2237897 & $K C N Q 1$ & $T H$ & 49 & 3,610 \\
\hline rs7961581 & & TSPAN8 & 51 & 3,635 \\
\hline rs864745 & $J A Z F 1$ & $T A X 1 B P 1$ & 101 & 5,108 \\
\hline rs10010131 & WFS1 & MRFAP1 & 138 & 5,504 \\
\hline rs564398 & & $C D K N 2 A$ & 145 & 5,565 \\
\hline rs10010131 & WFS1 & $C P Z$ & 286 & 6,449 \\
\hline $\begin{array}{l}\text { rs1111875 } \\
\text { rs5015480 }\end{array}$ & & TMEM20 & 347 & 6,701 \\
\hline rs 12779790 & & DHTKDI & 471 & 7,012 \\
\hline rs2237897 & $K C N Q 1$ & $S L C 22 A 18$ & 488 & 7,047 \\
\hline $\begin{array}{l}\text { rs } 4506565 \\
\text { rs } 7903146\end{array}$ & $T C F 7 L 2$ & DCLRE1A & 748 & 7,483 \\
\hline \multicolumn{5}{|l|}{ rs7901695 } \\
\hline rs10010131 & WFS1 & JAKMIP1 & 1,288 & 7,748 \\
\hline rs564398 & & PTPLAD2 & 1,409 & 7,774 \\
\hline rs2237892 & $K C N Q 1$ & $C A R S$ & 1,414 & 7,779 \\
\hline rs2237892 & $K C N Q 1$ & NAP1L4 & 1,955 & 7,864 \\
\hline rs7961581 & & TMEM19 & 2,243 & 7,842 \\
\hline rs4607103 & & PSMD6 & 2,630 & 7,790 \\
\hline $\begin{array}{l}\text { rs } 4506565 \\
\text { rs7901695 }\end{array}$ & $T C F 7 L 2$ & PDCD4 & 3,496 & 7,445 \\
\hline \multicolumn{5}{|l|}{ rs7903146 } \\
\hline rs2237892 & $K C N Q 1$ & $M R G P R E$ & 3,615 & 7,394 \\
\hline rs 13266634 & SLC30A8 & THRAPG & 4,001 & 7,211 \\
\hline rs7578597 & THADA & ZFP36L2 & 4,266 & 7,117 \\
\hline rs 12255372 & $T C F 7 L 2$ & $R B M 20$ & 4,870 & 6,788 \\
\hline rs864745 & $J A Z F 1$ & HOXА13 & 5,330 & 6,535 \\
\hline rs10010131 & WFS1 & STK32B & 5,457 & 6,470 \\
\hline rs 12779790 & & SEC61A2 & 7,895 & 4,828 \\
\hline
\end{tabular}

$\Delta$ Rank, the difference between type 2 diabetes coexpression rank and background rank; T2D coexpression rank, the rank of each gene's type 2 diabetes coexpression score 
Fig. 2 (a) Enrichment of $p_{\mathrm{T} 2 \mathrm{D}}<0.05$ at increasing type 2 diabetes coexpression score quantiles. The genes in each quantile were ordered by decreasing $\Delta$ rank, then split at the median $\Delta$ rank. The genes in each half quantile were connected to eSNPs using eQTL datasets mapped in liver, omental and subcutaneous adipose tissue. The blue and red points represent, respectively, the $p_{\mathrm{T} 2 \mathrm{D}}<0.05$ for the top and bottom halves. To estimate the null distribution, we randomly selected half of the genes in each group 10,000 times and observed the $p_{\text {T2D }}<0.05$ for each sample. The violin plots represent the distribution of the permutations for each set of genes. The separation between the top and bottom halves as the threshold is increased suggests that the coexpression rank, coupled with the $\Delta \mathrm{rank}$, is a useful metric for selecting genes relevant to the disease process. $p=0.12$ for 97.5 percentile; $p=0.007$ for 99 percentile. (b) Estimation of the null distribution by permuting the initial expression traits. As another estimate of the null distribution, we randomly selected 33 genes as the initial expression traits. As in (a), we then observed the $p_{\mathrm{T} 2 \mathrm{D}}<0.05$ in WTCCC data for the top half of genes at each cut-off for each sample. The violin plots represent the distribution of this value at each cut-off for each sample. We repeated this permutation analysis 1,000 times. $p=0.066$ for 97.5 percentile; $p=0.026$ for 99 percentile. (c) Validation of results using an independent dataset. To validate our findings shown in (b), we repeated the permutation analysis using data obtained from the GENEVA diabetes study. $p=0.13$ for 99.5 percentile; $p=0.042$ for 99.75 percentile. Blue diamond, $p_{\mathrm{T} 2 \mathrm{D}}<0.05$ for genes with change in rank greater than quantile median; red diamond, $p_{\mathrm{T} 2 \mathrm{D}}<0.05$ for genes with change in rank less than quantile median. T2D, type 2 diabetes

The genes in the 99th percentile cut-off that mapped to WTCCC are listed in Table 3. Eight of these genes have eSNPs with $p<0.05$ for type 2 diabetes. Two (HMGCS1, IDII) are involved in steroid synthesis and one (KHK) is involved in monosaccharide metabolism.

\section{Discussion}

GWASs are revealing increasing numbers of loci associated with various diseases. However, our understanding of the biological mechanisms behind these genetic variants is, in many cases, incomplete. eQTLs have the potential to aid in deciphering these variants by associating them with gene expression.

Although a recent study discovered no significant eQTL relationships for well-known type 2 diabetes SNPs in colon, pancreas or liver tissue [23], our initial analysis of type 2 diabetes expression traits revealed several such examples. This most likely reflects the large number of individuals from whom we could collect samples, as well as our inclusion of adipose tissue in this study. For instance, the expression of TCF7L2 is not associated with any of the SNPs studied, but there are several genes, including VTIIA, PDCD4 and CASP7, whose expression is associated with SNPs in TCF7L2. VTI1A, which we observed as an expression trait of the TCF7L2 SNP in omental adipose tissue, is a vesicle-soluble NSF attachment protein receptor (v-SNARE) that is a component of insulin-sensitive GLUT4-containing vesicles and affects insulin-dependent glucose transport in
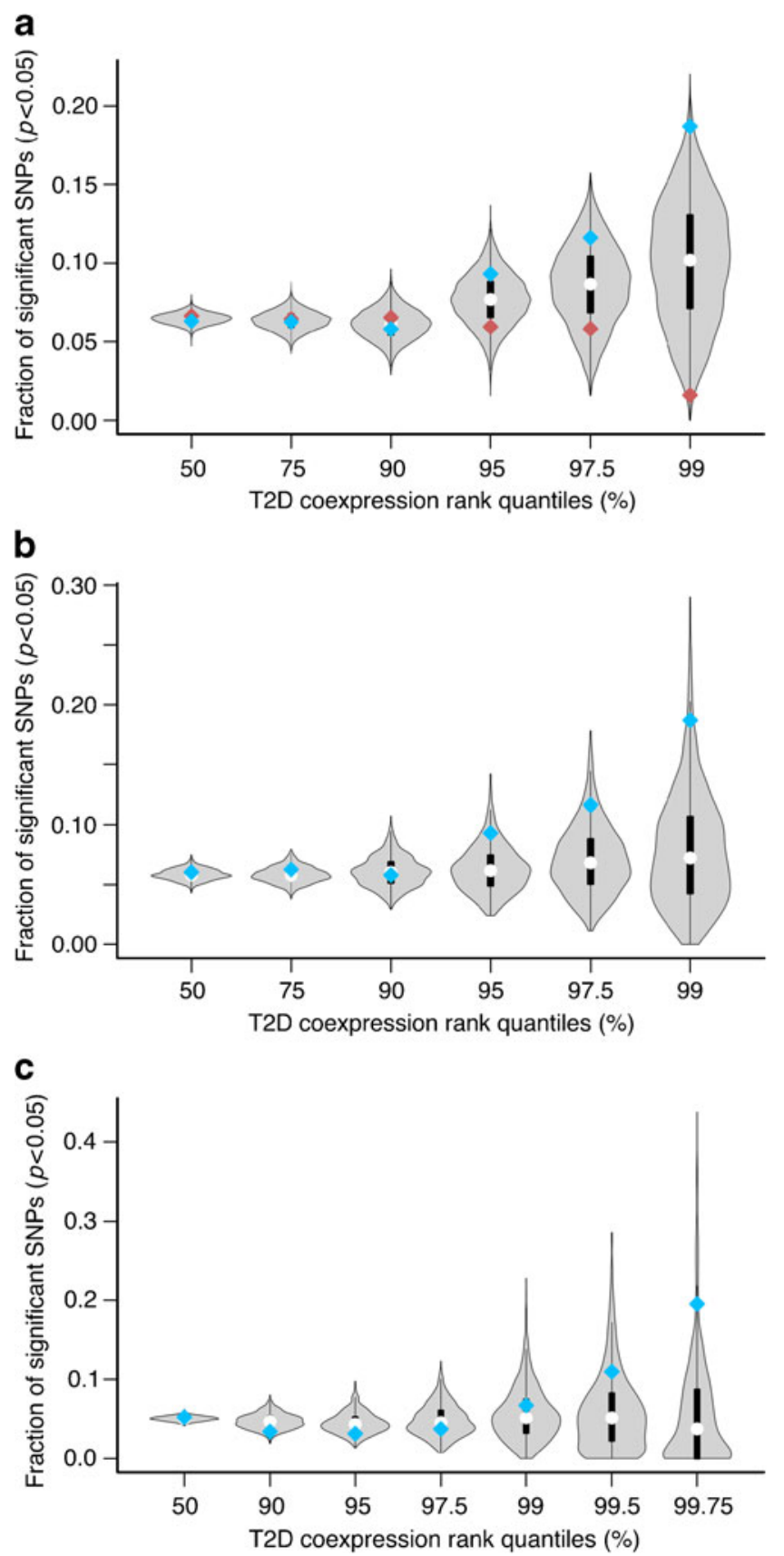

adipocytes [24]; $P D C D 4$, another expression trait of the same SNP, plays a crucial role in pancreatic beta cell death in type 1 diabetes [25]; CASP7 has also been identified as a positional candidate gene for type 1 diabetes [26]. All of these expression traits could serve as causal explanations of how the TCF7L2 SNP leads to type 2 diabetes.

As another example, rs1111875 and rs5015480, variants located close to the HHEX gene, are actually associated with the expression of $I D E$ (encoding insulin-degrading enzyme) in subcutaneous fat. This association suggests that the functional significance of these SNPs in type 2 diabetes is relayed through the expression of $I D E$, which plays a central 
Table 3 Top 30 candidate type 2 diabetes genes, out of 6,005 , with the selected type 2 diabetes expression traits with which they are coexpressed

\begin{tabular}{|c|c|c|c|c|}
\hline $\begin{array}{l}\text { Gene containing } \\
\text { T2D SNP }\end{array}$ & $\begin{array}{l}\text { Expression } \\
\text { trait }\end{array}$ & $\begin{array}{l}\text { Coexpressed } \\
\text { candidate gene }\end{array}$ & $\begin{array}{l}\text { Coexpression } \\
\text { rank }\end{array}$ & $\Delta$ Rank \\
\hline$T C F 7 L 2$ & $V T I 1 A$ & $T N N C 1$ & 14 & 1,492 \\
\hline TCF7L2 & CASP7 & KBTBD10 & 19 & 1,886 \\
\hline TCF7L2 & DCLRE1A & $P G A M 2$ & 21 & 1,960 \\
\hline$K C N Q 1$ & TNNT3 & CKMT2 & 32 & 2,020 \\
\hline WFS1 & $M R F A P 1$ & $H R C$ & 37 & 1,457 \\
\hline$K C N Q 1$ & NAP1L4 & $T X L N B$ & 38 & 1,230 \\
\hline SLC $30 A 8$ & THRAP6 & $T C A P^{\mathrm{a}}$ & 54 & 2,668 \\
\hline WFS1 & $C P Z$ & RYRI & 55 & 1,241 \\
\hline THADA & ZFP36L2 & $F R Y^{\mathrm{a}}$ & 60 & 2,624 \\
\hline$K C N Q 1$ & $K C N Q 1$ & LMOD2 & 63 & 1,465 \\
\hline$K C N Q 1$ & $C A R S$ & CACNAIS & 64 & 3,171 \\
\hline$J A Z F 1$ & $T A X 1 B P 1$ & $E C H D C 1$ & 65 & 3,189 \\
\hline TCF7L2 & PDCD4 & $N A V 2$ & 66 & 2,535 \\
\hline WFS1 & $S T K 32 B$ & TMPRSS4 & 67 & 1,798 \\
\hline$K C N Q 1$ & $T H$ & $M L F 1$ & 72 & 1,628 \\
\hline Intergenic & $C D K N 2 A$ & $K I A A 0323^{\mathrm{b}}$ & 78 & 1,850 \\
\hline$K C N Q 1$ & $S L C 22 A 18$ & ANKRD33 & 87 & 3,086 \\
\hline Intergenic & TSPAN8 & $P Y G M$ & 88 & 2,481 \\
\hline$K C N Q 1$ & $M R G P R E$ & $I D I 1^{\mathrm{a}}$ & 89 & 1,220 \\
\hline Intergenic & TMEM20 & MTHFDIL & 97 & 2,701 \\
\hline Intergenic & SEC61A2 & $H M G C S 1^{\mathrm{a}}$ & 98 & 1,927 \\
\hline Intergenic & PSMD6 & $C D 44^{\mathrm{a}}$ & 106 & 1,321 \\
\hline$T C F 7 L 2$ & $G P A M$ & $L M C D 1$ & 107 & 2,950 \\
\hline WFS1 & $J A K M I P 1$ & $P T G D R^{\mathrm{a}}$ & 109 & 4,974 \\
\hline$J A Z F 1$ & HOXA13 & $F A D S 2$ & 114 & 1,529 \\
\hline Intergenic & ADAMTS9 & $M Y O M 1^{\mathrm{a}}$ & 117 & 1,754 \\
\hline Intergenic & $I D E$ & PDLIM3 & 119 & 2,177 \\
\hline$T C F 7 L 2$ & CASP7 & INSIG1 & 120 & 1,529 \\
\hline$K C N Q 1$ & $K C N Q 1$ & PFKM & 121 & 2,902 \\
\hline Intergenic & PTPLAD2 & $K H K^{\mathrm{a}}$ & 124 & 2,722 \\
\hline
\end{tabular}

Coexpression rank, the rank of the type 2 diabetes coexpression score for each gene $\Delta$ Rank, the difference between type 2 diabetes coexpression rank and background rank

${ }^{a}$ Any linked eSNP with $p<0.05$ in WTCCC

${ }^{\mathrm{b}}$ Also known as KHNYN

T2D, type 2 diabetes

role in insulin metabolism [27]. In other words, explaining GWAS findings using eSNPs, as demonstrated here, might help to distinguish between two nearby genes with radically different potential mechanisms for disease.

Finally, rs564398, a variant in $C D K N 2 B$ antisense RNA 1 $(C D K N 2 B-A S 1)$, is associated with the expression of $C D K N 2 A$ in omental adipose tissue and PTPLAD2 in liver. A recent study reported that this SNP was associated with the expression of $C D K N 2 B-A S 1$ but not $C D K N 2 A / B$ in peripheral blood [28]; our result may be specific to the tissue types that we investigated. Here, our analysis using eSNPs suggests several different candidate mechanisms across separate tissues; it could be that higher-significance variants for type 2 diabetes play their role through distinct mechanisms in multiple relevant tissues.

In this study, we devised a ranking system that uses these type 2 diabetes expression traits in combination with coexpression networks in metabolically important tissues to discover novel genes associated with type 2 diabetes. By combining eQTL datasets from two different studies, we discovered that eSNPs regulating highly ranked genes in these tissues had a significant rising trend for association with type 2 diabetes in two well-known GWASs, specifically those performed by the WTCCC and GENEVA initiative. While the trend observed in the GENEVA study reached significance at a higher quantile cut-off, we think this is still sufficient to confirm our findings.

Having thus confirmed our hypothesis, we reasoned that the highly ranked genes that were not even marginally associated with type 2 diabetes in a GWAS might also be relevant to the pathogenesis of type 2 diabetes. We therefore investigated the other novel genes highlighted by our algorithm. Many of these novel genes that we identified are primarily expressed in skeletal or cardiac muscle. Several of these are involved in insulin signalling or glucose metabolism. For example, $\mathrm{Ca}^{2+}$ influx through L-type $\mathrm{Ca}^{2+}$ channels $(C A C N A 1 S)$ is essential for glucose-stimulated insulin secretion [29], while sarcosin (KBTBD10) is a cytoskeletal protein that, like $V T I 1 A$, is associated with the insulinstimulated glucose transporter GLUT4; interestingly, this association is suppressed in the presence of insulin [30]. PYGM (phosphorylase, glycogen, muscle) and PFKM (phosphofructokinase, muscle) are key enzymes in glycogenolysis and glycolysis.

We also discovered several genes involved in cholesterol (INSIG1, insulin induced gene 1; HMGCS1, HMG-CoA synthase $1 ; I D I 1$, isopentenyl diphosphate isomerase 1) and fatty acid (FADS2, fatty acid desaturase 2; ECHDC1, enoyl CoA hydratase domain containing 1) metabolism. In particular, FADS2 activity has been linked to the risk of developing type 2 diabetes [31].

Fructokinase $(K H K)$ is another interesting candidate gene revealed by our analysis. The endproduct of $K H K$ is fructose1-phosphate, which accelerates release of glucokinase $(G K)$ from its regulatory protein (GKRP) [32]. Glucokinase serves as an insulin sensor in the pancreatic beta cells and is being evaluated as a potential drug target for type 2 diabetes [33].

The method presented here represents a paradigm for using eQTLs and prior knowledge of SNPs associated with a disease to discover additional candidate genes and variants for that disease. The strength of this approach lies in the fact that it incorporates the functional significance of the SNPs 
encapsulated in the association of eQTLs and expression traits. In addition, using coexpression networks constructed in various tissues enables the discovery of candidate genes not expressed in the tissues used for mapping eQTLs. As the number and quality of tissue-specific eQTL studies increase and improve, we anticipate that the power of this type of analysis to detect novel associations will also be enhanced dramatically. This highlights once again the importance of making this type of data available, so that the greater community of scientists may benefit.

Acknowledgements We thank A. Skrenchuk and M. Seda from Stanford University for computer support.

Funding This work was supported by the Lucile Packard Foundation for Children's Health, the Hewlett Packard Foundation and the National Library of Medicine (R01 LM009719 and T15 LM007033). This study makes use of data generated by the WTCCC and GENEVA. A full list of the investigators who contributed to the generation of the data is available from www.wtccc.org.uk and www.genevastudy.org, respectively. These projects were funded by the Wellcome Trust under award 076113 and the National Human Genome Research Institute under award U01HG00439, respectively.

Duality of interest The authors declare that there is no duality of interest associated with this manuscript.

Contribution statement AJB and EES conceived the study. HPK, AJB and EES designed the study. All authors contributed to analysing and interpreting the data. HPK, EC, RC, and AJB drafted the article. HPK, AJB, EES, XY and BZ revised the article. All authors approved the final version.

Open Access This article is distributed under the terms of the Creative Commons Attribution License which permits any use, distribution, and reproduction in any medium, provided the original author(s) and the source are credited.

\section{References}

1. Manolio TA (2010) Genomewide association studies and assessment of the risk of disease. N Engl J Med 363:166-176

2. Bray NJ, Buckland PR, Owen MJ, O'Donovan MC (2003) Cis-acting variation in the expression of a high proportion of genes in human brain. Hum Genet 113:149-153

3. Stranger BE, Forrest MS, Clark AG et al (2005) Genome-wide associations of gene expression variation in humans. PLoS Genet 1:e78

4. Nicolae DL, Gamazon E, Zhang W, Duan S, Dolan ME, Cox NJ (2010) Trait-associated SNPs are more likely to be eQTLs: annotation to enhance discovery from GWAS. PLoS Genet 6:e1000888

5. Ding J, Gudjonsson JE, Liang L et al (2010) Gene expression in skin and lymphoblastoid cells: refined statistical method reveals extensive overlap in cis-eQTL signals. Am J Hum Genet 87:779-789

6. Richards AL, Jones L, Moskvina V et al (2012) Schizophrenia susceptibility alleles are enriched for alleles that affect gene expression in adult human brain. Molecular Psychiatry 17:193-201

7. Zhong H, Beaulaurier J, Lum PY et al (2010) Liver and adipose expression associated SNPs are enriched for association to type 2 diabetes. PLoS Genet 6:e1000932
8. Fransen K, Visschedijk MC, van Sommeren S et al (2010) Analysis of SNPs with an effect on gene expression identifies UBE2L3 and BCL3 as potential new risk genes for Crohn's disease. Hum Mol Genet 19:3482-3488

9. Chen Y, Zhu J, Lum PY et al (2008) Variations in DNA elucidate molecular networks that cause disease. Nature 452:429-435

10. Greenawalt DM, Dobrin R, Chudin E et al (2011) A survey of the genetics of stomach, liver, and adipose gene expression from a morbidly obese cohort. Genome Res 21:1008-1016

11. Schadt EE, Molony C, Chudin E et al (2008) Mapping the genetic architecture of gene expression in human liver. PLoS Biol 6:e107

12. Yang X, Zhang B, Molony C et al (2010) Systematic genetic and genomic analysis of cytochrome P450 enzyme activities in human liver. Genome Res 20:1020-1036

13. Emilsson V, Thorleifsson G, Zhang B et al (2008) Genetics of gene expression and its effect on disease. Nature 452:423-428

14. Schadt EE, Lamb J, Yang X et al (2005) An integrative genomics approach to infer causal associations between gene expression and disease. Nat Genet 37:710-717

15. Derry JMJ, Zhong H, Molony C et al (2010) Identification of genes and networks driving cardiovascular and metabolic phenotypes in a mouse F2 intercross. PLoS One 5:e14319

16. Zhang B, Horvath S (2005) A general framework for weighted gene co-expression network analysis. Stat Appl Genet Mol Biol; 4:article 17

17. Takeuchi F, Serizawa M, Yamamoto K et al (2009) Confirmation of multiple risk Loci and genetic impacts by a genome-wide association study of type 2 diabetes in the Japanese population. Diabetes 58:1690-1699

18. Hu C, Wang C, Zhang R et al (2009) Variations in KCNQ1 are associated with type 2 diabetes and beta cell function in a Chinese population. Diabetologia 52:1322-1325

19. Sale MM, Smith SG, Mychaleckyj JC et al (2007) Variants of the transcription factor 7-like 2 (TCF7L2) gene are associated with type 2 diabetes in an African-American population enriched for nephropathy. Diabetes 56:2638-2642

20. Saxena R, Gianniny L, Burtt NP et al (2006) Common single nucleotide polymorphisms in TCF7L2 are reproducibly associated with type 2 diabetes and reduce the insulin response to glucose in nondiabetic individuals. Diabetes 55:2890-2895

21. Voight BF, Scott LJ, Steinthorsdottir V et al (2010) Twelve type 2 diabetes susceptibility loci identified through large-scale association analysis. Nat Genet 42:579-589

22. Timpson NJ, Lindgren CM, Weedon MN et al (2009) Adiposityrelated heterogeneity in patterns of type 2 diabetes susceptibility observed in genome-wide association data. Diabetes 58:505-510

23. Cotsapas C, Prokunina-Olsson L, Welch C et al (2010) Expression analysis of loci associated with type 2 diabetes in human tissues. Diabetologia 53:2334-2339

24. Bose A (2005) The v-SNARE Vtila regulates insulin-stimulated glucose transport and Acrp30 secretion in 3T3-L1 adipocytes. J Biol Chem 280:36946-36951

25. Ruan Q, Wang T, Kameswaran V et al (2011) The microRNA-21PDCD 4 axis prevents type 1 diabetes by blocking pancreatic $\beta$ cell death. Proc Natl Acad Sci USA 108:12030-12035

26. Babu SR, Bao F, Roberts CM et al (2003) Caspase 7 is a positional candidate gene for IDDM 17 in a Bedouin Arab family. Ann NY Acad Sci 1005:340-343

27. Farris W (2003) Insulin-degrading enzyme regulates the levels of insulin, amyloid beta-protein, and the beta-amyloid precursor protein intracellular domain in vivo. Proc Natl Acad Sci 100:41624167

28. Cunnington MS, Koref MS, Mayosi BM, Burn J, Keavney B (2010) Chromosome 9p21 SNPs associated with multiple disease phenotypes correlate with ANRIL expression. PLoS Genet 6: e1000899 
29. Henquin JC (2000) Triggering and amplifying pathways of regulation of insulin secretion by glucose. Diabetes 49:1751-1760

30. Foster LJ, Rudich A, Talior I et al (2006) Insulin-dependent interactions of proteins with glut4 revealed through stable isotope labeling by amino acids in cell culture (SILAC). J Proteome Res 5:64-75

31. Kröger J, Zietemann V, Enzenbach C et al (2011) Erythrocyte membrane phospholipid fatty acids, desaturase activity, and dietary fatty acids in relation to risk of type 2 diabetes in the European
Prospective Investigation into Cancer and Nutrition (EPIC)-Potsdam Study. Am J Clin Nutr 93:127-142

32. Hayward BE, Fantes JA, Warner JP et al (1996) Colocalization of the ketohexokinase and glucokinase regulator genes to a $500-\mathrm{kb}$ region of chromosome $2 \mathrm{p} 23$. Mammalian Genome 7:454-458

33. Matschinsky FM (2009) Assessing the potential of glucokinase activators in diabetes therapy. Nat Rev Drug Discov $8: 399-416$ 